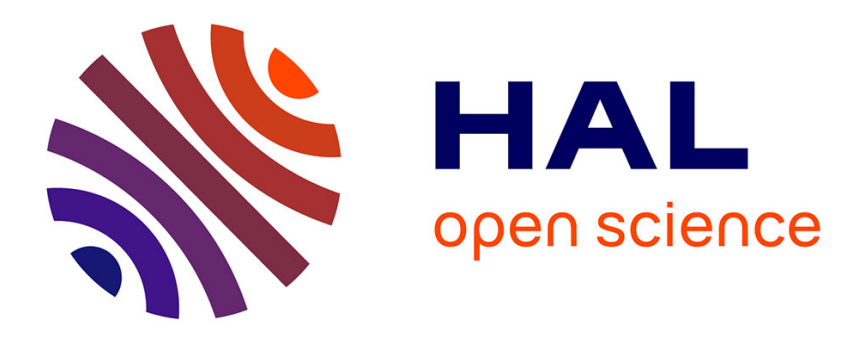

\title{
Les constructions existentielles en français, en espagnol et en italien
}

\author{
Machteld Meulleman
}

\section{To cite this version:}

Machteld Meulleman. Les constructions existentielles en français, en espagnol et en italien. XXVe Congrès international de linguistique et de philologie romanes, Sep 2007, Innsbrück, Autriche. pp.369378. hal-02471369

\author{
HAL Id: hal-02471369 \\ https://hal.science/hal-02471369
}

Submitted on 29 Mar 2021

HAL is a multi-disciplinary open access archive for the deposit and dissemination of scientific research documents, whether they are published or not. The documents may come from teaching and research institutions in France or abroad, or from public or private research centers.
L'archive ouverte pluridisciplinaire HAL, est destinée au dépôt et à la diffusion de documents scientifiques de niveau recherche, publiés ou non, émanant des établissements d'enseignement et de recherche français ou étrangers, des laboratoires publics ou privés. 


\section{Machteld Meulleman}

\section{Les constructions existentielles en français, en espagnol et en italien}

\section{Introduction}

Pour introduire des référents dans le discours, beaucoup de langues utilisent des constructions présentatives sur la base d'un verbe existentiel (Lambrecht 1994). Le français, l'espagnol et l'italien disposent à ce propos notamment de deux constructions:

(a) Les présentatifs existentiels (PE), à savoir il y a en français, hay en espagnol et $c$ 'è en italien, dans lesquels le verbe existentiel a dans une large mesure perdu sa valeur sémantique d'existence suite à un processus de grammaticalisation (Givón 2001);

(b) Le verbe existentiel intransitif (VEI) exister en français, existir en espagnol et esistere en italien avec postposition du sujet (soit par inversion, soit dans une construction impersonnelle).

En anglais les constructions existentielles (CE) introduites par there ont fait l'objet de nombreuses études, entre autres dans le cadre de la grammaire générative (cf. Lasnik 1992), relationnelle (cf. Levin / Rappaport 1995) et cognitive (cf. Lakoff 1987). Pour les langues romanes, la plupart des études se sont concentrées sur la fonction discursive des structures en il y a au niveau macro-syntaxique (cf. Lambrecht 1994) ou sur la grammaticalisation des différents présentatifs existentiels (cf. Blasco Ferrer 2004). La Fauci / Loporcaro (1997) présentent une approche comparative dans le cadre de la grammaire relationnelle. La structure argumentale des CE reste toutefois très controversée quant au statut du SN mais encore et surtout quant au statut syntaxique (argument ou adjoint) du locatif (cf. Conti Jiménez 2005; Fernández Soriano 1999; Treviño 2003).

A notre connaissance jusqu'à présent aucune étude comparative des deux constructions existentielles dans les trois langues romanes en question n'a été proposée. Dans cette contribution nous étudierons à partir d'une analyse empirique les différentes caractéristiques syntaxiques et distributionnelles des six verbes existentiels. ${ }^{1}$ Par la suite nous essaierons d'interpréter ces données et de voir notamment dans quelle mesure ces six constructions présentent une tendance plus ou moins prominente à la lexicalisation ou à la spécialisation pragmatico-discursive en tant qu'instruments présentatifs.

1 Notre analyse empirique se base sur un corpus journalistique écrit de 1000 occurrences par verbe étudié (6000 au total). Pour le français nous utilisons le corpus du Monde de 1994 et 1996, pour l'espagnol le corpus journalistique de 2001 et 2002 du Corpus de Referencia del Español Actual et pour l'italien un corpus constitué d'articles sortis de Il Mondo, Il Tempo et Avvenire de 2006 et 2007. 
Nous commencerons par un bref relevé des propriétés syntaxiques des constructions existentielles présentatives (CEP) $(\S 2)$, suivi de la présentation de notre analyse empirique des caractéristiques du SN postverbal (§3) et du locatif (\$4). Nous terminerons par une conclusion.

\section{Propriétés syntaxiques}

Une première différence importante entre les présentatifs existentiels dans les trois langues consiste en le choix du verbe existentiel. ${ }^{2}$ L'italien exprime l'existence par le verbe esserci et il y a accord entre le $\mathrm{V}$ et le SN postverbal. ${ }^{3}$ En revanche le français et l'espagnol utilisent une forme impersonnelle du verbe avoir et haber respectivement. ${ }^{4}$

Une deuxième caractéristique formelle intéressante est la présence d'une particule locative dans les trois présentatifs existentiels. Celle-ci est présente formellement dans tous les temps et tous les modes en français ( $y$ dans $i l y a$ ) et en italien ( $c i$ dans $c$ 'èlci sono), alors qu'en espagnol elle apparaît uniquement à l'indicatif présent hay sous la forme d'un $-y$ enclitique. Lorsqu'il y a un autre élément locatif présent dans la phrase existentielle, il y a donc toujours cooccurrence avec le pronom locatif. Aussi pour la langue actuelle ce pronom locatif est-il généralement considéré comme un locatif grammaticalisé qui n'a plus de fonction syntaxique et qui est devenu un élément formel caractérisant les présentatifs existentiels. $^{5}$

En revanche, pour les VEI exister, existir et esistere il n'y a jamais cooccurrence d'un locatif nominal et d'une particule locative. Lorsque le pronom locatif apparaît, il a toujours une fonction clairement anaphorique. Ainsi dans l'exemple (1) le pronom locatif y renvoie au SN les Etats-Unis dans la phrase précédente.

(1) Et les Etats-Unis, ce n'est pas la France. Il n'y existe ni politique sociale, ni politique de la ville, ni revenu minimum d'insertion. (Le Monde, 13/01/1995)

Ces divergences formelles entre les différentes constructions existentielles dans les trois langues, quant au choix du verbe existentiel et quant à la présence constante d'une particule locative, constituent le départ de notre analyse comparative. Il y a notamment deux questions qui se posent:

2 Le latin classique exprimait l'existence par esse, mais en latin vulgaire ce verbe a été substitué par habet, la forme impersonnelle du verbe habere. Le français et l'espagnol ont généralisé l'emploi de habet, alors que l'italien a continué à exprimer l'existence par esse. Ce choix était déjà assez clair en langue médiévale.

3 La variante avec averci (et avervi) n'est usitée que très rarement dans la langue courante.

4 L'emploi de il est en français est nettement moins fréquent et limité à des contextes littéraires.

5 Dans la langue médiévale il y avait une distribution complémentaire quasi parfaite entre la présence d'un locatif nominal et pronominal dans les trois langues. L'impossibilité de la cooccurrence des deux types de locatifs pourrait constituer un argument en faveur d'une analyse du locatif comme argumental dans la langue ancienne (La Fauci / Loporcaro 1997). 
1) Peut-on considérer il y a, hay et c'è d'un côté et exister, existir et esistere de l'autre comme des structures fonctionnellement équivalentes?

2) Y a-t-il des différences syntaxiques et fonctionnelles entre respectivement il y $a$ et $i l$ existe, hay et existe(n) et c'è et esiste/esistono?

Afin de pouvoir formuler une réponse à cette question nous étudierons d'abord dans le détail les différentes caractéristiques du SN postverbal et ensuite celles du locatif.

\section{Le SN postverbal}

\subsection{Statut discursif}

Il ressort de notre analyse empirique que la suite des verbes existentiels est le plus souvent constituée d'un SN faible (avec ou sans post-détermination). ${ }^{6}$

\begin{tabular}{|l|l|l|l|l|l|l|l|l|}
\hline \multicolumn{7}{|c|}{ Tableau 1: Distribution de la séquence existentielle selon sa forme. } \\
\hline & \multicolumn{2}{|c|}{ SN faible } & \multicolumn{2}{|c|}{ SN fort } & \multicolumn{2}{c|}{ pronom } & \multicolumn{2}{c|}{ total } \\
\hline & $\#$ & $\%$ & $\#$ & $\%$ & $\#$ & $\%$ & $\#$ & $\%$ \\
\hline il y a & 767 & $77 \%$ & 123 & $12 \%$ & 110 & $11 \%$ & 1000 & $100 \%$ \\
\hline hay & 836 & $84 \%$ & 6 & $0,5 \%$ & 158 & $16 \%$ & 1000 & $100 \%$ \\
\hline c'è & 650 & $65 \%$ & 254 & $25 \%$ & 95 & $10 \%$ & 1000 & $100 \%$ \\
\hline il existe & 910 & $91 \%$ & 32 & $3 \%$ & 58 & $6 \%$ & 1000 & $100 \%$ \\
\hline existir+SN & 830 & $83 \%$ & 152 & $15 \%$ & 18 & $2 \%$ & 1000 & $100 \%$ \\
\hline esistere+SN & 814 & $81 \%$ & 157 & $16 \%$ & 290 & $3 \%$ & 1000 & $100 \%$ \\
\hline
\end{tabular}

Ces données statistiques ne sont pas surprenantes, puisque les verbes existentiels représentent le contexte de la Restriction de définitude par excellence (cf. Leonetti 2008). ${ }^{7}$ En effet, les VE assument la fonction pragmatique d'introduire des référents dans le discours. Comme dans l'exemple suivant (2), il s'agit généralement de référents nouveaux (hearer-new dans la terminologie de Prince (1992)). ${ }^{8}$

(2) Lorsqu'on se remémore la négociation conventionnelle de 1989, il y a là, c'est certain, une avancée culturelle. (Le Monde, 05/01/1994)

6 Nous entendons par $S N$ faible les SN indéfinis, partitifs et avec article zéro. Par $S N$ fort nous entendons les SN définis, démonstratifs et possessifs et les noms propres.

7 Dans la littérature la Definiteness Restriction reste un sujet de controverse. Elle peut s'expliquer tant à partir d'une approche sémantique (cf. Milsark 1977) que pragmatique (cf. Prince 1992).

8 Prince (1992) considère un référent comme hearer-new lorsqu'il ne fait pas partie du fond commun des interlocuteurs au moment de l'énonciation. 
En revanche il y a une nette différence entre les $\mathrm{V}$ existentiels quant à leur faculté d'introduire des référents forts. Ainsi il y $a, c^{\prime} e^{9}$, existir et esistere peuvent parfaitement introduire des référents connus mais inactifs (unused referents) comme les noms propres (3), là où hay et il existe ne permettent pas ce type de suite. Lorsque ces deux dernières constructions introduisent des SN forts, il s'agit toujours de SN grammaticalement définis mais pragmatiquement nouveaux comme les superlatifs (4). En fait ceci n'est nullement étonnant puisqu'il s'agit de constructions impersonnelles, souvent mises en rapport avec la restriction de définitude (cf. Dobrovie-Sorin / Beyssade 2004). L'on pourrait donc dire que c'est plutôt la locution impersonnelle il y a qui fait bande à part en admettant l'introduction de référents connus mais inactifs.

(3) La chanteuse arrive en concierge, [...] Sous les oripeaux, il y a Catherine Ringer en robe courte jaune et noir et collants écossais. (Le Monde, 02/02/1994)

(4) Lo que le pido a este partido es que en las gradas haya la máxima euforia y el máximo apoyo a los jugadores. (Faro de Vigo, 15/06/2001)

Notons également que la séquence existentielle est deux fois plus souvent de nature pronominale derrière les présentatifs existentiels que derrière les VEI. Il s'agit alors de pronoms indéfinis comme quien dans l'exemple (5).

(5) Hay quien opina que una cultura subvencionada es una cultura sin futuro, pero la marginación histórica del gallego merece una reparación. (El Cultural, 17/10/2002)

\subsection{Distribution lexicale}

Notre analyse empirique relève également une différence dans la distribution lexicale des présentatifs existentiels et des verbes existentiels intransitifs. Voici les quatre noms postverbaux les plus fréquents en ordre décroissant pour les six verbes:

(a) il y a: chose, personne, gens, cas

(b) haber: persona, gente, diferencia, cosa

(c) c'è: tempo, persona, ragazza, differenza

(d) exister: solution, différence, politique, preuve

(e) existir: posibilidad, diferencia, problema, riesgo

(f) esistere: possibilità, differenza, prodotto, versione

Les référents les plus fréquents derrière les présentatifs existentiels sont d'une part des lexèmes très vagues tels que chose ou personne et d'autre part des lexèmes abstraits tels que différence. Il est remarquable que les référents des SN les plus fréquents derrière les

9 La fréquence relativement haute de $\mathrm{SN}$ forts derrière c'è a été souvent discutée dans la littérature. Selon Moro (1997) le présentatif italien n'est pas sujet à la restriction de définitude, l'italien étant une langue à sujet zéro. Toutefois pour d'autres auteurs la restriction s'applique également dans les CEP italiennes, mais selon d'autres paramètres (Leonetti 2008). 
VEI soient tous du second type, alors que le premier type de lexème est extrêmement rare. Cette différence distributionnelle correspond à celle observée pour les pronoms indéfinis.

Cette observation pourrait indiquer une légère tendance vers une spécialisation lexicale partielle parallèle des deux types de constructions existentielles dans les trois langues, tant pour les pronoms que pour les SN.

\section{Le locatif}

Passons maintenant aux différentes caractéristiques des locatifs dans la CEP. Tout d'abord nous dirons quelques mots sur la présence de locatifs dans les CEP. Ensuite nous passerons à la position de ces locatifs, et cela en fonction de leur forme morphosyntaxique.

\subsection{Ancrage locatif}

Voici les données statistiques pour la présence de locatifs dans la CEP:

\begin{tabular}{|l|c|c|c|c|c|c|}
\hline \multicolumn{7}{|c|}{ Tableau 2: Présence de locatifs dans la CEP. } \\
\hline & \multicolumn{2}{|c|}{ LOC + } & \multicolumn{1}{c|}{$\varnothing$} & \multicolumn{2}{c|}{ total } \\
\hline & $\#$ & $\%$ & $\#$ & $\%$ & $\#$ & $\%$ \\
\hline ilya & 508 & $51 \%$ & 492 & $49 \%$ & 1000 & $100 \%$ \\
\hline hay & 447 & $45 \%$ & 553 & $55 \%$ & 1000 & $100 \%$ \\
\hline$c^{\prime}$ 'è & 493 & $49 \%$ & 507 & $51 \%$ & 1000 & $100 \%$ \\
\hline il existe & 487 & $49 \%$ & 513 & $51 \%$ & 1000 & $100 \%$ \\
\hline existir+SN & 464 & $46 \%$ & 536 & $54 \%$ & 1000 & $100 \%$ \\
\hline esistere+SN & 442 & $44 \%$ & 558 & $56 \%$ & 1000 & $100 \%$ \\
\hline
\end{tabular}

Il est remarquable que dans près de la moitié des occurrences il y ait absence de locatif dans les deux types de constructions existentielles et cela dans les trois langues. Toutefois dans ce cas le SN postverbal est souvent modifié par une relative restrictive classant le SN antécédent dans une sous-classe de son genre. Ainsi l'on pourrait paraphraser l'exemple suivant (6) comme parmi tous les trains, il y en a aussi qui arrivent à l'heure.

(6) Loin des polémiques qui agitent les chancelleries, des discussions sur le retrait de l'ONU de Bosnie, le général Cot souligne qu'il y a aussi des trains qui arrivent à l'heure, des succès de la Forpronu qui sont souvent méconnus. (Le Monde, 01/02/1994)

Ici il s'agit en réalité d'une relation partie-tout où le tout ou la classe peut s'exprimer par un locatif. L'absence de locatif explicite dans ces exemples n'infirme donc pas 
nécessairement l'hypothèse locative ${ }^{10}$, puisqu'elle peut s'expliquer par l'existence d'un ensemble-localisateur implicite souvent associé à ce type de phrases existentielles (Furukawa 1996; Van De Velde 2005).

\subsection{Position des locatifs}

Quant à la position des locatifs dans les CEP, trois cas de figure se présentent:

1) le locatif est initial, il se trouve devant le verbe existentiel et le $\mathrm{SN}$

2) le locatif est intercalé, il se trouve entre le verbe existentiel et le SN

3) le locatif est final, il se trouve derrière le verbe existentiel et le SN.

La catégorie grammaticale des locatifs pouvant influer sur leur position dans la CEP, nous opérons une distinction entre les SP et les adverbes locatifs.

Comme le montre le tableau suivant, la position du SP locatif varie nettement selon les langues.

\begin{tabular}{|l|l|l|l|l|l|l|l|l|}
\hline \multicolumn{7}{|c|}{ Tableau 3: La position des locatifs SP dans la CEP. } \\
\hline \multicolumn{1}{|c|}{ LOC = SP } & Locatif initial & \multicolumn{2}{|c|}{ Locatif intercalé } & \multicolumn{2}{c|}{ Locatif final } & \multicolumn{2}{c|}{ total } \\
\hline & $\#$ & $\%$ & $\#$ & $\%$ & $\#$ & $\%$ & $\#$ & $\%$ \\
\hline ily a & 166 & $41 \%$ & 81 & $20 \%$ & 159 & $39 \%$ & 406 & $100 \%$ \\
\hline hay & 229 & $62 \%$ & 18 & $5 \%$ & 122 & $33 \%$ & 369 & $100 \%$ \\
\hline c'è & 354 & $83 \%$ & 12 & $3 \%$ & 61 & $14 \%$ & 427 & $100 \%$ \\
\hline il existe & 152 & $38 \%$ & 111 & $28 \%$ & 136 & $34 \%$ & 399 & $100 \%$ \\
\hline existir+SN & 276 & $65 \%$ & 24 & $6 \%$ & 122 & $29 \%$ & 422 & $100 \%$ \\
\hline esistere+SN & 182 & $63 \%$ & 7 & $2 \%$ & 102 & $35 \%$ & 291 & $100 \%$ \\
\hline
\end{tabular}

Là où les SP locatifs se trouvent de préférence en position initiale en espagnol et en italien (exemple 7), aussi bien pour ce qui est des présentatifs existentiels que des VEI, le français divise les SP locatifs préverbaux entre la position initiale et intercalée (exemple 8). Le locatif SP apparaît en position finale dans environ un tiers des cas dans les trois langues, sauf auprès du présentatif italien c'è qui semble mettre les locatifs SP systématiquement en position initiale.

(7) Fidel mi ha assicurato: posso morire, ma a Cuba c'è una squadra ed un popolo pronti a fare andare avanti la Rivoluzione. (Il Tempo, 14/08/2006)

(8) Il y a en Bosnie entre 1500 et 2000 volontaires de Croatie. (Le Monde, 01/02/1994)

En étudiant ces occurrences de plus près, on constate qu'il ne semble pas y avoir de restriction sur le type de SP locatif intercalé en français, ni quant au type d'ancrage locatif (spatial ou temporel), ni quant à la longueur. En effet il n'est pas rare de trouver des

10 La locative hypothesis considère les constructions existentielles comme des constructions locatives (cf. Clark 1978; Freeze 1992). 
occurrences où il y a intercalation de deux locatifs (spatial et temporel) comme dans l'exemple (9). Il semble donc que, même si la différence est moins nette, il y a se comporte comme exister quant à la position des SP locatifs, en se distinguant ainsi des quatre autres verbes existentiels.

(9) Comment traiter avec la Russie? Il y a eu pendant longtemps dans l'entourage du président Clinton la croyance un peu naïve qu'une Russie réformiste et démocratique serait par nature un partenaire facile et prévisible. (Le Monde, 26/02/1994)

Comme le montre le tableau suivant, la différence entre les langues est encore plus nette lorsque le locatif est un adverbe.

\begin{tabular}{|l|l|l|l|l|l|l|l|l|}
\hline \multicolumn{8}{|c|}{ Tableau 4: La position des locatifs adverbiaux dans la CEP. } \\
\hline LOC = ADV & Locatif initial & \multicolumn{2}{|c|}{ Locatif intercalé } & \multicolumn{2}{|c|}{ Locatif final } & \multicolumn{2}{c|}{ total } \\
\hline & $\#$ & $\%$ & $\#$ & $\%$ & $\#$ & $\%$ & $\#$ & $\%$ \\
\hline il y a & 26 & $25 \%$ & 62 & $61 \%$ & 14 & $14 \%$ & 102 & $100 \%$ \\
\hline hay & 61 & $78 \%$ & 9 & $12 \%$ & 8 & $10 \%$ & 78 & $100 \%$ \\
\hline c'è & 47 & $71 \%$ & 15 & $23 \%$ & 4 & $6 \%$ & 66 & $100 \%$ \\
\hline il existe & 10 & $11 \%$ & 78 & $89 \%$ & 0 & $0 \%$ & 88 & $100 \%$ \\
\hline existir+SN & 33 & $79 \%$ & 9 & $21 \%$ & 0 & $0 \%$ & 42 & $100 \%$ \\
\hline esistere+SN & 37 & $73 \%$ & 14 & $27 \%$ & 0 & $0 \%$ & 51 & $100 \%$ \\
\hline
\end{tabular}

En espagnol et en italien l'adverbe locatif se trouve dans la grande majorité des cas en position initiale comme dans l'exemple (10). En revanche, en français il se trouve le plus souvent en position intercalée, tant pour le présentatif il y a (exemple 11) que pour le VEI $i l$ existe.

(10) Ma perché questo strano fenomeno di una notte dello spirito che dura praticamente tutta la vita? Qui c'è qualcosa di nuovo rispetto a quello che hanno vissuto e spiegato i maestri del passato, [...]. (Avvenire, 26/08/2007)

(11) $[\ldots]$, le CNPF avait dû faire des concessions qui n'étaient pas compatibles avec les exigences de la compétition. Il y a là une contradiction qui n'est pas soutenable. (Le Monde, $15 / 01 / 1994)$

L'analyse comparée de la position des locatifs dans la phrase existentielle montre que même si la fréquence d'un ancrage spatio-temporel ne diffère pas pour les six verbes existentiels, cet ancrage n'occupe pas la même position dans les trois langues, le français admettant plus facilement l'intercalation des SP locatifs et préférant même cette position pour les adverbes locatifs.

Comment donc expliquer cette différence de position? A notre avis il y a dans les phrases existentielles présentatives un conflit entre la pression syntaxique de l'ordre SVO 
et le principe thématique qui veut que le thème précède au rhème. Le locatif ${ }^{11}$ désignant le cadre spatio-temporel dans lequel apparaît le nouveau référant est un thème idéal et devrait donc se trouver de préférence en position initiale. C'est ce qui se présente en espagnol et en italien. En revanche en français la pression syntaxique de l'ordre SVO semble l'emporter sur la pression thématique, puisqu'un sujet explétif $i l$ apparaît en position initale et que le locatif suit généralement le SN.

La différence de position des locatifs en français d'une part et en espagnol et en italien d'autre part pourrait donc être due à des caractéristiques syntaxiques propres aux langues en question et non spécifiques à la construction existentielle. Cette problématique n'étant pas dans le centre d'intérêt de cet article, nous n'approfondirons pas ici cette question pourtant très intéressante (cf. Roegiest / Meulleman 2005).

\section{Conclusion}

Si les deux constructions existentielles dans les trois langues servent grosso modo un but communicatif commun, en l'occurrence l'introduction de référents dans le discours, elles présentent néanmoins d'importantes différences.

D'une part les présentatifs existentiels il y $a$, hay et c'è se distinguent des verbes existentiels intransitifs exister, existir et esistere par une possible spécialisation lexicale partielle différente quant au type de référent introduit. Les PE introduisent en effet nettement plus souvent des référents plus vagues que les VEP, tant sous la forme de pronoms indéfinis que sous la forme de $\mathrm{SN}$ tels que chose, gente, personne. Les VEI de leur part introduisent presque exclusivement des référents abstraits.

D'autre part les constructions existentielles hay et il existe s'opposent aux autres CEP par un respect plus strict de la Restriction de définitude. Il pourrait donc y avoir une spécialisation pragmatico-discursive quant au statut discursif (connu / inactif / nouveau) du SN introduit dans le discours. Cette question complexe mérite une analyse plus détaillée, notamment quant au statut sémantique du SN introduit (cf. la notion de spécificité).

Finalement les constructions existentielles françaises il $y$ a et il existe diffèrent des constructions existentielles espagnoles et italiennes par la position des locatifs dans la CEP. Là où l'espagnol et l'italien préfèrent mettre les locatifs en position initiale, le français intercale un nombre important de locatifs entre le verbe existentiel et le référent introduit.

Nous estimons que ces différences syntaxiques et distributionnelles pourraient en partie s'expliquer par des différences formelles quant au choix du verbe existentiel et son emploi impersonnel ou non. Toutefois les différences constatées nous amènent avant tout à de nouvelles questions, notamment concernant d'éventuelles implications au niveau argumental. Y a-t-il un locatif présent dans la structure argumentale des deux types de

11 Les CEP sont généralement considérées comme des constructions thétiques, qui par définition ne contiennent pas de thème (cf. Lambrecht 1994). D'autres auteurs affirment toutefois que dans les constructions existentielles le locatif assume toujours le rôle de thème, même s'il reste implicite (cf. Leonetti 2008). 
constructions? S'il en est ainsi, assume-t-il la même fonction? Et pourquoi est-il absent dans près de la moitié des occurrences? Toutes ces questions feront l'objet de nos futures recherches.

\section{Bibliographie}

Blasco Ferrer, Eduardo (2004): Tipologia, storia e classificazione delle costruzioni presentative romanze: contributo a una teoria della grammaticalizzazione. In: Quaderns de Filologia. Estudis lingüístics 9, 27-49.

Conti Jiménez, Carmen (2005): Existential sentences with preposed locative phrases and postverbal determinerless subjects in Spanish. In: Linguistics 46, 6, 1079-1104.

Clark, Eve Vivienne (1978): Locationals: existential, locative and possessive constructions. In: Greenberg, Joseph (ed.): Universals of human language. Vol. 4: Syntax. Stanford / California: Stanford University Press, 85-126.

Dobrovie-Sorin, Carmen / Beyssade, Claire (2004): Définir les indéfinis. Paris: CNRS Editions.

Fernández Soriano, Olga (1999): Two types of impersonal sentences in Spanish: locative and dative subjects. In: Syntax 2, 2, 101-140.

Freeze, Ray (1992): Existentials and other locatives. In: Language 48, 3, 552-595.

Furukawa, Naoyo (1996): Grammaire de la prédication seconde. Forme, sens et contraintes. Louvain-la-Neuve: Duculot.

Givón, Talmy (2001): Syntax: an introduction. Amsterdam / Philadelphia: John Benjamins.

La Fauci, Nunzio / Loporcaro, Michele (1997): Outline of a theory of existentials on evidence from Romance. In: SILTA 26, 1, 5-55.

Lakoff, George (1987): Women, fire and dangerous things: what categories reveal about the mind. Chicago: UCP.

Lambrecht, Knud (1994): Information structure and sentence form. Cambridge: Cambridge University Press.

Lasnik, Howard (1992): Case and expletives: notes toward a parametric account. In: LingI 23, 381405.

Leonetti, Manuel (2008): Definiteness effects and the role of the coda in existential constructions. In: Klinge, Alex / Hoeg-Muller, Hendrik (edd.): Essays on nominal determiniation. Amsterdam: John Benjamins.

Levin, Beth / Rappaport Hovav, Malka (1995): Unaccusativity: at the syntax-lexical semantics interface. Cambridge (Massachusetts): MIT-press.

Milsark, Gary (1977): Toward an explanation of certain pecularities in the existential construction in English. In: Linguistic Analysis 3, 1-29.

Moro, Andrea (1997): The raising of predicates. Cambridge: Cambridge University Press.

Prince, Ellen (1992): The ZPG-letter: Subjects, definiteness, and information status. In: Thompson, Sandra / Mann, William (edd.): Discourse description: Diverse linguistic analyses of a fund raising text. Philadelphia: John Benjamins, 295-325.

Roegiest, Eugeen / Meulleman, Machteld (2005): La structure thématique de la phrase existentielle en français et en espagnol. In: RomGG 11, 2, 157-172.

Treviño, Esthela (2003): On the subjecthood issue of existential haber. In: Montrul, Silvina / Ordoñez, Francisco: Linguistic theory and language development in Hispanic languages. Somerville: Cascadilla Press, 178-192.

Van De Velde, Danièle (2005): Les interprétations partitive et existentielle des indéfinis dans les phrases existentielles locatives. In: TraLing 50, 37-52. 
